\section{Apt screening with microfluidics}

\section{By Michael J. Haas, Senior Writer}

California researchers have developed a microfluidics-based platform that isolated nucleic acid aptamer leads with nanomolar binding affinities in a single round of screening. Although the paper provided proof of principle by screening a DNA aptamer library, companies polled by SciBX think the technology could also be used to screen libraries of small molecules and peptides.

Nucleic acid aptamers are single strands of DNA or RNA that can bind to and inhibit proteins and other molecular targets. The current method of screening aptamer libraries-systematic evolution of ligands by exposure enrichment (SELEX) - uses molecular separation techniques to select for hits and PCR to amplify those hits prior to the next round of screening.

A typical aptamer library has $10^{14} \mathrm{mem}$ bers, which is several orders of magnitude more than the phage display libraries used to screen for antibodies. As a result, SELEX usu-

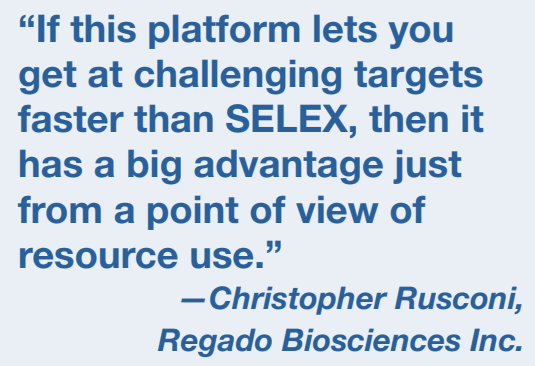

magnetic bead-assisted SELEX methods with microfluidics technology that allowed for automated handling and selection of microscale quantities of both the bead-immobilized target and the library-which conventional SELEX cannot do.

They described their platform-microfluidic SELEX (M-SELEX) -and provided proof-of-concept results with the target protein light chain recombinant Botulinum neurotoxin A in a paper in the Proceedings of the National Academy of Sciences. ${ }^{1}$

After a single screening round of a $10^{14}$-member aptamer library, the team selected 15 aptamers and found 4 with neurotoxin A binding affinities of 34-86 nM.

The team wrote that the platform should be extendable to other screening applications "ranging from small molecules and proteins to viral and cellular surface proteins" because the technology depends more on microfluidic physics than the chemistry of the chosen target.

The group was led by $\mathrm{H}$. Tom Soh, associate professor of mechanical engineering and materials at UCSB, and Alan Heeger, professor of physics and materials at UCSB. Neither was available for comment.

\section{Need for speed}

Although companies contacted by SciBX agreed that M-SELEX could screen aptamer libraries faster than SELEX, they differed on whether this would be a meaningful advance in aptamer discovery.

Christopher Rusconi, CSO of Regado Biosciences Inc., said the platform overcomes a ally requires eight or more rounds of screening to isolate aptamers with nanomolar affinities. Phage display usually takes three or four rounds.

SELEX was developed by a research team at the University of Colorado at Boulder. Team leader Larry Gold, professor of molecular, cellular and developmental biology, founded NeXagen Inc. in 1991 to commercialize the technology. In 1995 NeXagen and Vestar Inc. merged to become NeXstar Inc., which Gilead Sciences Inc. acquired in 1999. Gilead exclusively licensed SELEX to Archemix Corp. in 2001.

In 2005 Macugen pegaptanib, a pegylated nucleic acid aptamer that targets VEGF, became the first therapeutic aptamer approved for use in the U.S. The compound, discovered at Gilead, is now marketed by Eyetech Inc. and Pfizer Inc. to treat wet age-related macular degeneration (AMD).

According to Archemix, SELEX can isolate high-binding aptamers in 5-15 rounds of screening-about one month's work.

A team from the University of California, Santa Barbara and Lawrence Livermore National Laboratory has sought to decrease screening rounds by employing a key fact about binding interactions: the smallest amount of target will yield hits with the highest binding affinities in a given round of screening. The researchers combined key limitation of SELEX by increasing the odds of isolating library compounds with the highest affinity.

"You can more purely select for binding," he said. "Hopefully the aptamers are more selective for what you want-target binding — and not just winners in the system used."

Typically, Rusconi said, each round of screening increases the possibility of selecting molecules that perform well on the technology being applied-for instance, molecules that replicate and amplify well-instead of culling out just those with the highest binding affinity for the target.

"Other combinatorial technologies face this same problem, but the sheer size of an aptamer library makes it a greater concern with SELEX," Rusconi said. "If this platform lets you get at challenging targets faster than SELEX, then it has a big advantage just from a point of view of resource use."

Regado's aptamer-based REG1 anticoagulation system is in a Phase IIa trial as anticoagulation therapy in acute coronary syndrome (ACS) patients undergoing coronary revascularization procedures. The system is composed of RB006, a single-stranded nucleic acid aptamer that binds to and inhibits Factor IXa, and RB007, a complementary nucleic acid aptamer that binds to and neutralizes RB006.

Page Bouchard, SVP of discovery and preclinical development at 
Archemix, agreed that M-SELEX could speed up the early stage of aptamer discovery. However, Bouchard doesn't think SELEX itself is a research bottleneck, and as a result he expects that the new platform will not significantly improve lead discovery times.

Instead, he said any new technology would have to demonstrate improvement over SELEX in one or two key areas: target hit rate or the binding affinities of the isolated aptamers. "Any process that can improve upon either of these
"Ideally we would like to see better affinities demonstrated for targets for which there are known aptamers, shown side-byside in a standard binding assay."

-Page Bouchard, Archemix Corp.
"These researchers can probably use this platform so well because they are material scientists and engineers" with expertise and equipment that the typical molecular biologist lacks, he said. "So if a biologist wanted to think about adopting this platform, it would be hard to go out and give it a whirl."

By comparison, SELEX uses common molecular biology techniques that make it usable by almost anyone, he said.

But Rusconi added that M-SELEX could parameters would be of great interest from a commercial perspective," but the platform reported in PNAS does not appear to improve either parameter, he said.

In January, Archemix began a Phase IIb trial of ARC1779, an antiplatelet aptamer that targets von Willebrand Factor (vWF) to treat thrombotic microangiopathies.

\section{Target practice}

Questions of speed aside, there was consensus that M-SELEX needs to be validated against other targets to prove its commercial viability.

Bouchard pointed out that single stranded DNA aptamers with single-digit nanomolar binding affinities for Botulinum neurotoxin A-the PNAS team's target-have already been reported. ${ }^{2}$ Thus, he said, "more M-SELEX will have to be done against more targets to make a convincing case. Ideally we would like to see better affinities demonstrated for targets for which there are known aptamers, shown side-by-side in a standard binding assay."

Regado's Rusconi agreed that the platform should be "test driven against benchmarks where it's known what binding affinity makes for a good aptamer against that target" to allow for a direct comparison with SELEX. Thrombin or VEGF would be good choices, he said.

Rusconi added that the team's choice of target also made it impossible to know whether more than one round of screening would be required to isolate aptamers with nanomolar affinities against benchmark targets. Finally, he said the platform would likely require specialized equipment and technical capabilities. have broader use than its predecessor because using "high concentrations and tiny volumes" should apply to any type of library or protein target.

Bouchard agreed. "The technology could in principle be used for any in vitro selection technique such as phage display, mRNA display or ribosome display," he said.

Sherylle Mills Englander, director of the office of technology and industry alliances at UCSB, told SciBX that she should be contacted directly by parties interested in the IP status of the Soh team's findings.

Haas, M.J. SciBX 2(7); doi:10.1038/scibx.2009.259

Published online Feb. 19, 2009

\section{REFERENCES}

1. Lou, X. et al. Proc. Natl. Acad. Sci. USA; published online Feb. 2, 2009; doi:10.1073/pnas.0813135106

Contact: H. Tom Soh, University of California, Santa Barbara, Calif. e-mail: tsoh@engineering.ucsb.edu

2. Tok, J. \& Fischer, N. Chem. Commun. (Camb.) 28, 1883-1885 (2008)

\section{COMPANIES AND INSTITUTIONS MENTIONED}

Archemix Corp., Cambridge, Mass.

Gilead Sciences Inc. (NASDAQ:GILD), Foster City, Calif.

Eyetech Inc., New York, N.Y.

Pfizer Inc. (NYSE:PFE), New York, N.Y.

Regado Biosciences Inc., Basking Ridge, N.J.

University of California, Santa Barbara, Calif.

University of Colorado at Boulder, Boulder, Colo.
Lawrence Livermore National Laboratory, Livermore, Calif. 